\title{
Steep and Adjustable Transfer Functions of Monolithic SOA-EA 2R-Regenerators
}

\author{
Öhman, Filip; Kjær, Rasmus; Christiansen, Lotte Jin; Yvind, Kresten; Mørk, Jesper
}

Published in:

I E E E Photonics Technology Letters

Link to article, DOI:

10.1109/LPT.2006.873359

Publication date:

2006

Document Version

Publisher's PDF, also known as Version of record

Link back to DTU Orbit

Citation (APA):

Öhman, F., Kjær, R., Christiansen, L. J., Yvind, K., \& Mørk, J. (2006). Steep and Adjustable Transfer Functions of Monolithic SOA-EA 2R-Regenerators. I E E E Photonics Technology Letters, 18(9), 1067-1069. https://doi.org/10.1109/LPT.2006.873359

\section{General rights}

Copyright and moral rights for the publications made accessible in the public portal are retained by the authors and/or other copyright owners and it is a condition of accessing publications that users recognise and abide by the legal requirements associated with these rights.

- Users may download and print one copy of any publication from the public portal for the purpose of private study or research.

- You may not further distribute the material or use it for any profit-making activity or commercial gain

- You may freely distribute the URL identifying the publication in the public portal 


\title{
Steep and Adjustable Transfer Functions of Monolithic SOA-EA 2R Regenerators
}

\author{
Filip Öhman, Rasmus Kjær, Lotte Jin Christiansen, Kresten Yvind, and Jesper Mørk
}

\begin{abstract}
Measurements and numerical modeling of a reamplification and reshaping $(2 \mathrm{R})$ regenerator demonstrate a steep power transfer function with adjustable threshold. The threshold can be adjusted more than $6 \mathrm{~dB}$ by simple control of the reverse bias voltage of the absorber section. The device consists of a semiconductor waveguide with alternating amplifier and absorber sections using quantum-well active material. The steep nonlinearity of the transfer function is achieved by concatenating several sections. We identify the saturation properties of the absorbing media, as dictated by the band-filling and field screening, as important for the observed transfer functions. The relation of the saturation powers of the gain and absorption sections is important for design optimization.
\end{abstract}

Index Terms-Noise, optical communication, optical signal processing, semiconductor optical amplifiers (SOAs).

\section{INTRODUCTION}

$\mathbf{I}$ $\mathrm{N}$ OPTICAL communication systems, there are several sources of signal degradation. Some impairments, such as attenuation and dispersion can be remedied by specific methods, e.g., amplification and dispersion compensation. However, random sources of signal degradation, like noise from amplifiers, require more general methods of signal restoration. All-optical reamplification and reshaping (2R) regeneration implies the improvement of the signal-to-noise ratio by, for example, extinction ratio (ER) improvement and noise redistribution. These improvements are achieved by a component with a nonlinear intensity transfer function [1], where the limit of a step function is equivalent to making a decision between a logical one and zero. A simple 2R-regenerator can be achieved by using the effect of saturable absorption in an electroabsorber (EA) [2]. The saturable absorber, however, suffers from high insertion losses and does not regenerate high intensity ("1") bits. This drawback can be remedied by combining the saturable absorber with a section of saturable gain in the form of a semiconductor optical amplifier (SOA) [3]. We have earlier proposed [4], [5] an integrated component for $2 \mathrm{R}$ regeneration consisting of an SOA and an EA and concatenated sections thereof, as shown in Fig. 1. These devices offer a very simple structure for $2 \mathrm{R}$-regeneration.

The important properties of the intensity transfer function are large attainable ER, strong nonlinearity, and the possibility to

Manuscript received November 30, 2005; revised February 15, 2006. This work was supported by the European Network of Excellence ePIXnet and by the Danish Technical Research Council through the SCOOP Program.

The authors are with COM DTU Department of Communications, Optics and Materials, Nano DTU, Technical University of Denmark, DK-2800 Kgs. Lyngby, Denmark (e-mail: fo@com.dtu.dk).

Digital Object Identifier 10.1109/LPT.2006.873359

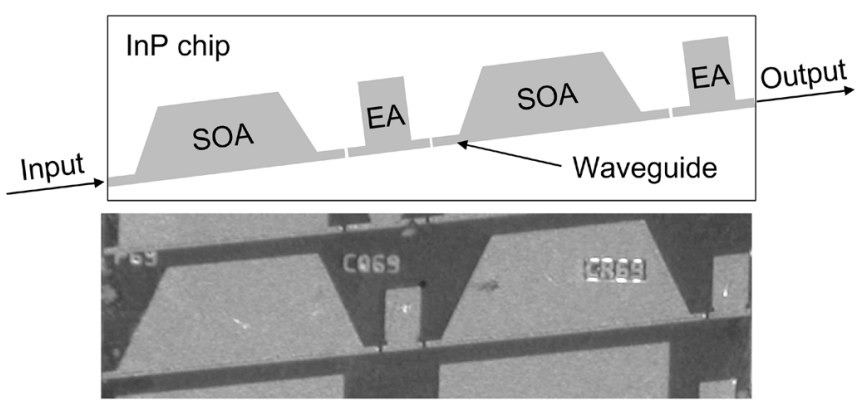

Fig. 1. Schematic and top view photo of the examined device consisting of two concatenated SOA-EA sections.

adjust the power threshold that separates the one- and zerolevels [6]. In this letter, we demonstrate experimentally that these properties are achieved in the SOA-EA regenerator by concatenating several amplifying and absorbing sections and by using proper bias conditions. We further show that these properties can be easily adjusted by changing the external bias. The results are also supported by a theoretical model, which is used for drawing important conclusions regarding design optimization.

\section{Device AND Numerical Model}

The examined device, shown in Fig. 1, consists of a single waveguide with a number of separate electrodes defining the amplifier and absorber sections, respectively. The waveguide is angled $7^{\circ}$ relative to the cleaved facets, which are also anti reflection coated to reduce reflections into the waveguide. The length of the SOA and EA sections are 545 and $120 \mu \mathrm{m}$, respectively. The active material consists of five 7.0-nm-thick compressively strained InGaAsP quantum wells in a strain compensated structure. The SOA gain peak is at $1535 \mathrm{~nm}$ and the $3-\mathrm{dB}$ optical bandwidth is roughly $60 \mathrm{~nm}$.

The SOA model used is a bidirectional model where the device is split into sections of equal length. In each section, the carrier density is assumed to be spatially constant and the section gain is calculated using a standard expression for semiconductor quantum-well material [7]. Carrier dynamics are governed by rate equations for the carrier density in each section. The rate equation takes into account the current-injection of carriers as well as depletion of carriers due to stimulated and spontaneous emission and nonradiative recombination. In the model, we found the inclusion of amplified spontaneous emission (ASE) to be of crucial importance for reproducing the behavior of the unsaturated SOA. The reason for this is that the 


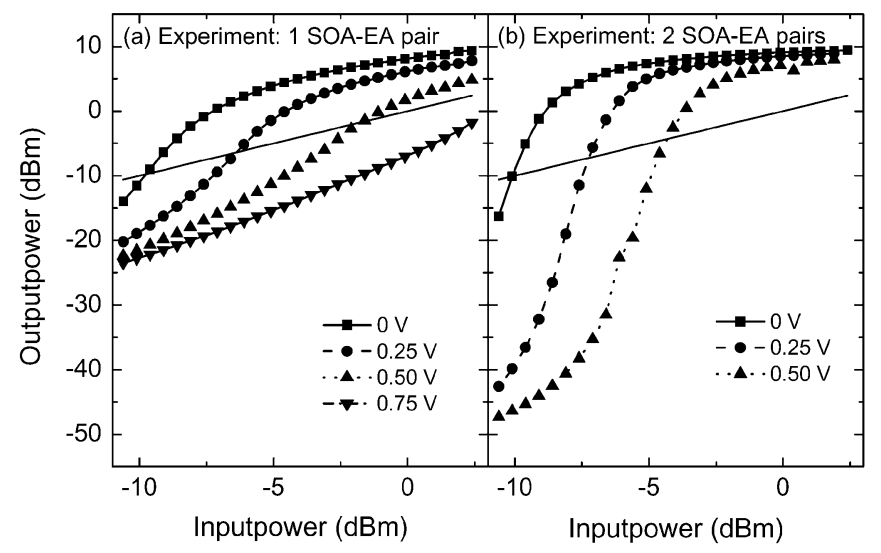

Fig. 2. Measured transfer functions for a single SOA-EA section (left) and two concatenated SOA-EA sections (right). The bias current to the SOA is $100 \mathrm{~mA}$ and the reverse bias of the first EA is varied, the second EA is unbiased. The straight line is the transfer function for a gain of $0 \mathrm{~dB}$.

small-signal gain of the SOA is limited by the ASE, which effectively prevents the small-signal gain from increasing to unrealistic values. Numerically, the ASE is represented as spectral densities with fixed frequency spacing. Bandgap shrinkage due to many-body effects is included using a numerical fit [9]. This effect causes the gain peak to red-shift as the bands become filled.

The EA absorption model is similar to the gain model used for the SOA with the exception of modifications including the effect of bandgap reduction due to the Franz-Keldysh effect and dependence of the carrier sweep-out time on the carrier density and reverse bias. The latter two are included phenomenologically [8], [9] and fitted to the experimental data. The carrier sweep-out time is assumed to be inversely proportional to the electric field across the quantum wells and the field is assumed to be reduced due to carrier screening by an amount proportional to the carrier density. All in all, these assumptions amount to a carrier density dependent sweep-out time $\tau$ so $(N)$ given by

$$
\tau_{\mathrm{so}}(N)=\frac{\tau_{0} \tau_{\mathrm{tr}}}{\tau_{\mathrm{tr}}\left(1-N / N_{\mathrm{tr}}\right)+\tau_{0} N / N_{\mathrm{tr}}}
$$

where $\tau_{0}$ is the sweep-out time with no optical input and $\tau_{\operatorname{tr}}$ and $N_{\text {tr }}$ are the sweep-out time and carrier density at transparency. Both $\tau_{0}$ and $\tau_{\text {tr }}$ are assumed to decrease exponentially with the external bias voltage [10] and are fitted to experimental data.

\section{RESUlTS AND DiSCUSSION}

The measured transfer functions of devices with one and two SOA-EA pairs are shown in Fig. 2(a) and (b), respectively. The wavelength is $1500 \mathrm{~nm}$, the current through each SOA section is $100 \mathrm{~mA}$, and the bias of the first EA section is varied between 0 and $-0.75 \mathrm{~V}$. The second EA is in this case unbiased. This is, however, not a general requirement for the proper operation of the device. The values for input and output power are the estimated value just inside the facets of the components. The coupling losses are estimate to $3 \mathrm{~dB}$.

It is clearly seen that the concatenation of an additional pair gives a stronger nonlinearity with a larger ER and threshold steepness, i.e., a large change in output power for a small change in input power at the regenerator threshold, i.e., the point where

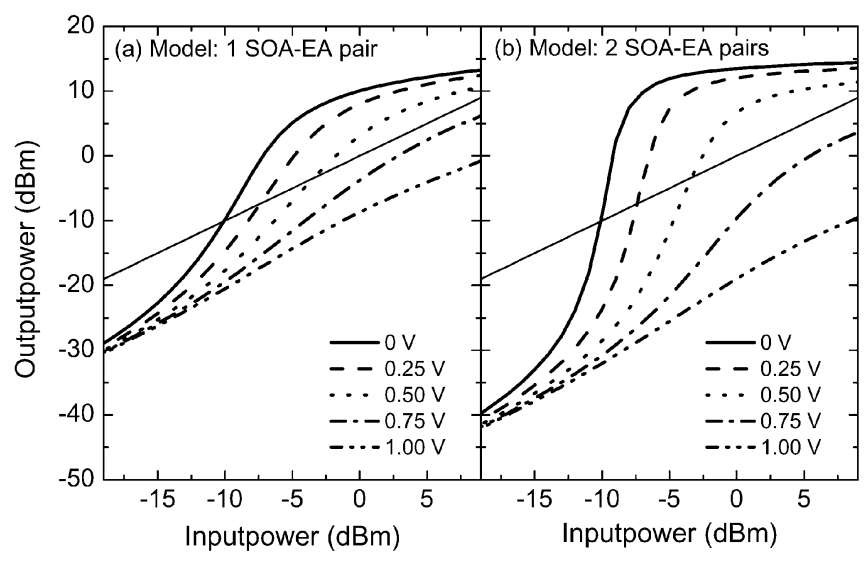

Fig. 3. Calculated transfer functions for a single SOA-EA section (left) and two concatenated SOA-EA sections (right). The straight line is the transfer function for a gain of $0 \mathrm{~dB}$.

the output power is the same as the input power. This is illustrated in Figs. 2 and 3 by a line showing the one-to-one relation. It should be noted that the additional gain section also introduces more noise, and hence, an increase in the noise figure [5]. In order to reduce this effect, the first SOA in the device should have as large a gain as possible [11]. Furthermore, the increase in nonlinearity can, if large enough, compensate for the higher noise figure by improving the redistribution of noise [6], [12]. In Fig. 2, the possibility to adjust the threshold of the regenerator using only the external bias on the absorber section is demonstrated. In both components, the input power threshold is seen to change with approximately $6 \mathrm{~dB}$ as the EA reverse bias is increased by $0.5 \mathrm{~V}$. In the one-pair case, there is also a reduction of nonlinearity at larger reverse bias, which will be discussed later in this section.

Fig. 3 shows simulated results using the model outlined in Section II. The values of the main parameters are the same as in the experiments except the SOA current, which is reduced to $72 \mathrm{~mA}$ in order to account for leakage current. The relevant EA parameter values are: $N_{\mathrm{tr}}=3.7 \times 10^{16} \mathrm{~m}^{-2}, \tau_{0}\left(V_{\mathrm{EA}}=\right.$ $0)=64$ ps and $\tau_{\mathrm{tr}}\left(V_{\mathrm{EA}}=0\right)=180$ ps. First of all, it can be concluded that the agreement between the model and experiment is good, and that the model shows the same behavior as the measurements when the reverse bias on the EA is changed. A reduction in nonlinearity for increasing reverse bias is seen in both the one-pair and the two-pair case.

The saturation power of the EA is the parameter mainly responsible for the shift of the threshold with reverse bias. The saturation of the EA is due to band filling and screening of the electrical field by the free carriers [13]. The main effect of a larger reverse bias is a reduction of the carrier sweep-out time, and hence, an increase of the saturation power $\left(P_{\text {sat }} \sim 1 / \tau_{\text {so }}\right)$ in the EA and a shift toward higher powers for the threshold of the SOA-EA transfer function, as seen in Figs. 2 and 3. In Fig. 4, the model is used for investigating the behavior of the transfer functions under different sweep-out conditions. In the left panel, the transfer function is plotted for different ratios of the sweep-out time at transparency and the sweep-out time without optical excitation $\left(\tau_{\operatorname{tr}} / \tau_{0}\right)$, i.e., the cases with maximum and no screening, respectively. This ratio is denoted 


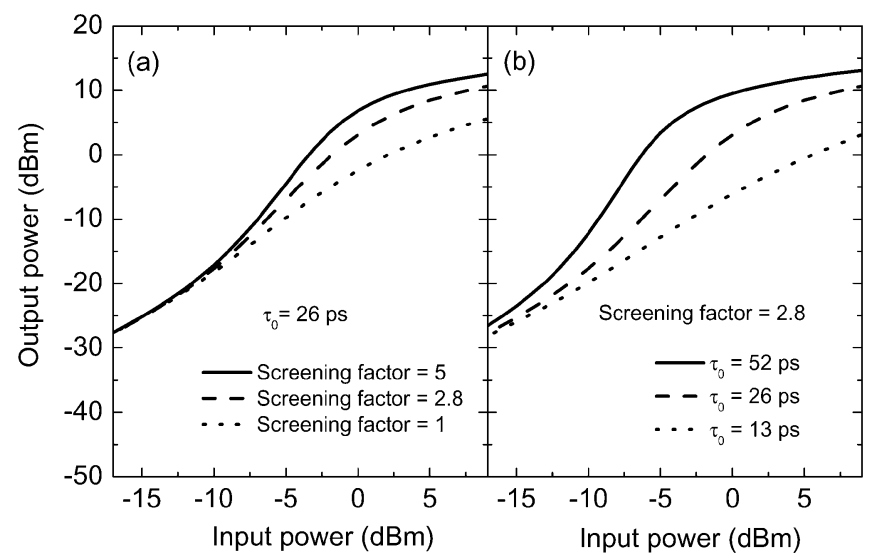

Fig. 4. Calculated transfer functions for one SOA-EA pair at a reverse bias of $0.5 \mathrm{~V}$ for (a) different screening factors $\left(\tau_{\mathrm{tr}} / \tau_{0}\right)$ and (b) sweep-out times.

the "screening factor" in the following and in the case of one SOA-EA pair, a value of 2.8 is found to give good agreement with experimental data. This value is used in Fig. 3.

It is clearly seen that when the effect of field screening is disregarded (i.e., when the effective sweep-out time is constant), the EA saturates at a slower rate with increasing input power and the nonlinear transfer function is less sharp. With a strong screening, the excitation of carriers leads to slower sweep-out, and hence, a stronger saturation and a sharper transfer function. It should be noted that a slow sweep-out also reduces the attainable speed of the device, but as long as the sweep-out is sufficiently fast, the absorber recovery time will not limit the attainable operation speed. The sweep-out time without optical excitation is 26 ps in all three cases of Fig. 4(a).

The relation of the saturation powers of the EA and SOA is also important. The saturation power is here defined as the power where the absorption or gain is half of the unsaturated value. If the EA has an input saturation power comparable to or higher than the output saturation power of the SOA, the absorber will not saturate sufficiently. This is the effect responsible for the steepness reduction at higher reverse bias in Fig. 3 and is demonstrated in Fig. 4(b), where the sweep-out time at zero optical injection is varied for a constant screening factor of 2.8. The ratio of the input saturation power of the EA to the output saturation power of the SOA in the three cases is $0.4,0.2$, and 0.1 , respectively. The SOA output saturation power is in the simulation fixed at $12 \mathrm{dBm}$ while the EA saturation power is varied by assuming different sweep-out times. For fast sweep-out time, the EA does not saturate at power levels below the output saturation power of the SOA and it is not possible to saturate the EA. In order to adjust the regenerator threshold to higher power levels, the output saturation power of the SOA has to be adjusted along with the input saturation power of the EA, for example by increasing the bias current. Fig. 4(b) shows the importance of designing the SOA with a sufficiently high saturation power, for example by using fewer quantum wells [14]. If, on the other hand, a better sensitivity, i.e., a lower minimum regenerator threshold, is wanted, a higher SOA gain and/or a lower saturation power of the EA is required. In the device used in this work, the active material is the same for both the gain and absorption sections. If additional growth steps are used in the fabrication [4], it is possible to use different active materials, which gives additional freedom when designing the bandgaps and saturation properties of the different sections.

\section{CONCLUSION}

The static transfer functions of a 2R-regenerator consisting of concatenated sections of saturable gain and absorption have a nonlinearity that can be increased by concatenating several SOA-EA pairs. By changing the bias conditions to the different sections, the saturation properties can be controlled and the threshold value adjusted in a simple way. A numerical model shows good agreement with the experiments and is used to investigate the influence of the effective carrier sweep-out time in the EA on the saturation properties. The fast sweep out time at high reverse bias increases the input saturation power of the EA, and hence, reduces the saturation of the EA and the sharpness of the transfer function. The screening of the electrical field in the EA by excited carriers, on the other hand, improves the saturation characteristics of the EA. The connection between sweep-out time and saturation leads to a tradeoff between operation speed and achievable nonlinearity.

\section{REFERENCES}

[1] P. Öhlen and E. Berglind, "Noise accumulation and BER estimates in concatenated nonlinear optoelectronic repeaters," IEEE Photon. Technol. Lett., vol. 9, no. 7, pp. 1011-1013, Jul. 1997.

[2] M. Tsurusawa et al., "Demonstration of optical noise reduction using nonlinear absorption in a semiconductor laser amplifier,' IEEE. J. Sel. Topics Quantum Electron., vol. 5, no. 3, pp. 861-865, May/Jun. 1999.

[3] C. Knöll et al., "Optimization of signal transmission by an in-line semiconductor optical amplifier-saturable absorber module," Opt. Commun., vol. 187, pp. 141-153, 2001.

[4] L. J. Christiansen et al., " $2 \mathrm{R}$ regeneration in concatenated semiconductor optical amplifiers and electroabsorbers," in Proc. ECOC 2004, Stockholm, Sweden, 2004, Paper Mo3.4.3.

[5] F. Öhman et al., "Noise and regeneration in semiconductor waveguides with saturable gain and absorption," IEEE J. Quantum Electron., vol. 40, no. 3, pp. 245-255, Mar. 2004.

[6] F. Öhman and J. Mørk, "Modeling of bit error rate in cascaded 2r regenerators," J. Lightw. Technol, vol. 24, no. 2, pp. 1057-1063, Feb. 2006

[7] L. A. Coldren and S. W. Corzine, Diode Lasers and Photonic Integrated Circuits, 1st ed. New York: Wiley, 1995.

[8] S. Højfeldt et al., "All-optical wavelength conversion and signal regeneration using an electroabsorption modulator," J. Lightw. Technol., vol. 18 , no. 8, pp. 1121-1127, Aug. 2000.

[9] S. Bischoff et al., "Monolithic colliding pulse mode-locked semiconductor lasers," Quantum Semiclass. Opt., vol. 9, pp. 655-674, 1997.

[10] J. Mulet et al., "Mode-locking dynamics in electrically driven vertical-external-cavity surface-emitting lasers," IEEE J. Quantum Electron., vol. 41, no. 9, pp. 1148-1156, Sep. 2004.

[11] D. M. Baney et al., "Theory and measurement techniques for the noise figure of optical amplifiers," Opt. Fiber Technol., vol. 6, pp. 122-154, 2000.

[12] J. Mørk et al., "Analytical expression for the bit-error-rate of cascaded all-optical regenerators," IEEE Photon. Technol. Lett., vol. 15, no. 10, pp. 1479-1481, Oct. 2003.

[13] S. Højfeldt and J. Mork, "Modeling of carrier dynamics in quantum-well electroabsorption modulators," IEEE. J. Sel. Topics Quantum Electron., vol. 8, no. 6, pp. 1265-1276, Nov./Dec. 2002.

[14] K. Yvind et al., "Low-jitter and high-power 40-GHz all-active modelocked lasers," IEEE Photon. Technol. Lett., vol. 16, no. 4, pp. 975-977, Apr. 2004. 